\title{
Probing insulin bioactivity in oral nanoparticles produced by ultrasonication-assisted emulsification/internal gelation
}

This article was published in the following Dove Press journal:

International Journal of Nanomedicine

18 September 2015

Number of times this article has been viewed

Marlene A Lopes, ${ }^{1,2, *}$

Bárbara Abrahim-Vieira, ${ }^{3, *}$ Claudia Oliveira, ${ }^{4,5}$ Pedro Fonte, ${ }^{6,7}$ Alessandra M T Souza, ${ }^{3}$ Tammy Lira, ${ }^{3}$ Joana

A D Sequeira, ${ }^{1,2}$ Carlos

R Rodrigues, ${ }^{3}$ Lúcio $M$ Cabral, ${ }^{3}$ Bruno Sarmento, ${ }^{6-8}$ Raquel Seiça, ${ }^{9}$ Francisco Veiga, ${ }^{1,2}$ António J Ribeiro ${ }^{1,4,5}$

'Faculty of Pharmacy, University of Coimbra, Coimbra, Portugal; ${ }^{2} \mathrm{CNC}$ Center for Neuroscience and Cell Biology, Coimbra, Portugal; ${ }^{3}$ Department of Pharmaceutics, Faculty of Pharmacy, Federal University of Rio de Janeiro, Rio de Janeiro, Brazil; ${ }^{4} \mid 3 S$, Instituto de Investigação e Inovação em Saúde, University of Porto, Porto, Portugal; ${ }^{5}$ Group Genetics of Cognitive Dysfunction, IBMC - Instituto de Biologia Molecular e Celular, Porto, Portugal; 'REQUIMTE, Department of Chemical Sciences - Applied Chemistry Lab, Faculty of Pharmacy, University of Porto, Porto, Portugal; ${ }^{7}$ CESPU, Instituto de Investigação e Formação Avançada em Ciências e Tecnologias da Saúde, Gandra, Portugal; ${ }^{8}$ INEB - Instituto de Engenharia Biomédica, University of Porto, Porto, Portugal; ' IBILI - Institute of Biomedical Research in Light and Image, Faculty of Medicine, University of Coimbra, Coimbra, Portugal

*These authors contributed equally to this work

Correspondence: António J Ribeiro Faculty of Pharmacy, University of Coimbra, Azinhaga de Santa Comba 3000-548 Coimbra, Portugal

$\mathrm{Tel}+35$ I 239488414

Fax +35I 239488503

Email aribeiro@ff.uc.pt
Abstract: Alginate-dextran sulfate-based particles obtained by emulsification/internal gelation technology can be considered suitable carriers for oral insulin delivery. A rational study focused on the emulsification and particle recovery steps was developed in order to reduce particles to the nanosize range while keeping insulin bioactivity. There was a decrease in size when ultrasonication was used during emulsification, which was more pronounced when a cosurfactant was added. Ultrasonication add-on after particle recovery decreased aggregation and led to a narrower nanoscale particle-size distribution. Insulin encapsulation efficiency was $99.3 \% \pm 0.5 \%$, attributed to the strong $\mathrm{pH}$-stabilizing electrostatic effect between insulin and nanoparticle matrix polymers. Interactions between these polymers and insulin were predicted using molecular modeling studies through quantum mechanics calculations that allowed for prediction of the interaction model. In vitro release studies indicated well-preserved integrity of nanoparticles in simulated gastric fluid. Circular dichroism spectroscopy proved conformational stability of insulin and Fourier transform infrared spectroscopy technique showed rearrangements of insulin structure during processing. Moreover, in vivo biological activity in diabetic rats revealed no statistical difference when compared to nonencapsulated insulin, demonstrating retention of insulin activity. Our results demonstrate that alginate-dextran sulfate-based nanoparticles efficiently stabilize the loaded protein structure, presenting good physical properties for oral delivery of insulin.

Keywords: biopolymers, insulin secondary structure, microparticle, molecular modeling, nanoencapsulation processing, oral delivery

\section{Introduction}

The challenge of orally administered insulin has been addressed in order to help relieve the pain and stress caused during insulin injections by millions of diabetics. Insulin is a peptide drug and therefore it is a challenge for oral delivery, due to enzymatic degradation in the gastrointestinal tract and, particularly, to the difficulty to permeate the intestinal epithelium.

Much research has been focused on an oral insulin delivery system that mimics the physiologic pattern of insulin secretion. ${ }^{1-4}$ To ensure enteric protection, recent advances in nanoencapsulation resulted in an increasing growth of innovative formulations such as micro- ${ }^{5,6}$ and nanoparticles. ${ }^{7,8}$ In drug delivery, nanoparticles have demonstrated numerous advantages over conventional formulations. ${ }^{9,10}$ Particle size plays a crucial role in biomedical applications, namely on biorecognition, ${ }^{11}$ biodistribution, ${ }^{12}$ bioadhesion, ${ }^{13}$ biocompatibility, ${ }^{14}$ and biodegradation. ${ }^{15}$ However, most techniques for producing submicron particles require organic solvents, high temperature, or vigorous agitation, which are potentially harmful parameters to the 
structure of insulin and consequently lead to loss of activity. Submicron particles have been produced under particularly mild processing conditions by using emulsification/internal gelation technology, which has previously been applied to the production of larger-sized microparticles ${ }^{16-19}$ and micron- and nanometer-sized particles. ${ }^{20}$ This technology involves experimental conditions such as nonextreme values of temperature and $\mathrm{pH}$ and refraining from using harsh chemicals. All of those conditions are potentially threats to the stability of peptides and proteins. Indeed, this technology makes use of naturally occurring polymers such as alginate and dextran sulfate, which are a suitable choice due to their excellent biocompatibility, drug carrying ability, adjustable controlled-release property, lower cost, abundance in nature, and easier application. ${ }^{21}$ Previous studies revealed that the presence of a copolymer, such as dextran sulfate, enhances the loading of hydrophilic drugs and that the type of polymer has a considerable effect on the loading capacity. ${ }^{22}$

However, one of the limitations of emulsification/internal gelation technology consists of the difficulty to obtain monodispersed populations of particles. As drug-delivery systems, the size control and size uniformity of carriers are of great interest to generate in vitro and in vivo reproducible as well as repeatable profiles and behaviors, which will further result in benefits in clinical use. ${ }^{23}$ Alginate/dextran sulfate particles show polydispersed populations, characterized by a wide size distribution which derives from nano- and microparticles and also aggregates coexistence, ${ }^{24}$ characteristic of emulsification methods using biodegradable polymers. ${ }^{25}$ Over the past years, emulsification-based technologies have been optimized to produce polysaccharide-based particles with a lower size and monodispersed populations..$^{20,26-28}$ Ultrasonication is particularly effective in breaking up the aggregates of particles, thereby reducing their size and polydispersity. ${ }^{29}$ However, ultrasonication has also been related to formation of protein aggregates with structural changes. ${ }^{30}$

As delivery systems for insulin, the most important characteristics of particles include size distribution, encapsulation efficiency (EE), and the release profile of insulin from particles while maintaining the biological activity of insulin during and after processing. From an industrial and economic perspective, the EE is crucial especially in the case of proteins, which are expensive products. ${ }^{31}$

Stability is a major challenge especially when insulin is encapsulated in polymeric particles using an emulsion-based technique. The emulsification step is identified as critical for protein inactivation and aggregation. The hydrophobic interface created between the water and oily solvent, and the hydrophobic interactions between protein and polymer, may lead to adsorption of insulin at the interface, which results in insulin unfolding and/or aggregation. ${ }^{32-34}$ In addition, the shear used for the emulsification can also contribute to insulin denaturation. ${ }^{35}$ Hence, maintaining the native conformation of insulin during the encapsulation process is highly important, which otherwise may lead to protein aggregation and immunogenicity.

The purpose of this work was to develop a formulation of insulin-loaded biopolymer-based nanoparticles produced by emulsification/internal gelation technique with good physical properties for oral delivery and assess how emulsion droplet size may affect the resultant particles, while probing the activity of insulin after each step of the process. The emulsification step was performed with and without ultrasonication assistance and in the presence of one or two surfactants. The recovery strategy of particles was also investigated to increase recovery yield and ensure removal of residual oil from the surface of particles. For better insight about the formation of alginate/dextran sulfate particles, molecular modeling studies were performed. Here, quantum mechanics calculations were employed to understand the electronic properties of the multicomponent system of alginate/dextran sulfate.

Particles were characterized according to size distribution, surface charge, morphology, EE, and in vitro insulin release behavior. Activity of insulin during and after each stage of processing was assessed either in vitro or in vivo.

\section{Materials and methods Materials}

Low-viscosity sodium alginate (viscosity of $1 \%$ solution at $25^{\circ} \mathrm{C}, 4-12 \mathrm{cP}$ ), dextran sulfate, sorbitan monooleate $\left(\right.$ Span $\left.80^{\circledR}\right)$, phosphotungstic acid, trifluoroacetic acid 99\%, and acetonitrile (LiChrosolv) high-performance liquid chromatography (HPLC) grade were all obtained from Sigma-Aldrich Co. (St Louis, MO, USA); calcium carbonate (Setacarb; Omya, Orgon, France), paraffin oil (Scharlau, Barcelona, Spain), insulin 100 IU/mL (Actrapid ${ }^{\circledR}$; Novo Nordisk A/S, Bagsværd, Denmark), poloxamer 188 (Lutrol ${ }^{\circledR}$ F68; BASF, Ludwigshafen, Germany), sodium citrate dehydrate (Sigma-Aldrich Co., St Louis, MO, USA), and Nile red (Sigma-Aldrich Co.) were also obtained.

\section{Preparation of alginate/dextran sulfate- based particles}

Insulin-loaded particles were prepared through a modified emulsification/internal gelation technique. ${ }^{20,24}$ Briefly, 
the water in oil (w/o) emulsion was formed by mixing alginate/dextran sulfate matrix with the oil phase. An aqueous solution of sodium alginate $(2.0 \%, \mathrm{w} / \mathrm{v})$ and dextran sulfate $(0.75 \%, \mathrm{w} / \mathrm{v})$ was prepared by stirring $(100 \mathrm{rpm})$ overnight. Insulin was added and dissolved (100 IU/mL, $10 \mathrm{~mL})$. Then, an aqueous suspension of ultrafine calcium carbonate $(5 \%$, $\mathrm{w} / \mathrm{v}$ ) was ultrasonicated for 30 minutes to break up crystal aggregates, and was dispersed at a calcium-alginate ratio of $16.7 \%(\mathrm{w} / \mathrm{w})$. The resultant dispersion was emulsified within paraffin oil (aqueous phase/oil phase 50/50, v/v) facilitated by sorbitan monooleate $(2 \%, \mathrm{v} / \mathrm{v})$ by impeller-stirring homogenization (1,600 rpm). The effects of cosurfactant addition, poloxamer 188 , keeping the hydrophilic-lipophilic balance (HLB) required for a w/o emulsion (HLB $=4-6)^{36}$ and ultrasonication add-on during the emulsification step at different times $(10,15,20,25$, and 30 minutes) as well as at different ultrasonic amplitudes $(30 \%, 45 \%$, and $60 \%)$ were tested.

After emulsification, gelation was induced by addition of paraffin oil containing glacial acetic acid (molar ratio of acid-calcium, 3.5) to solubilize calcium dispersed in the alginate/dextran droplets for 15 minutes. Unloaded particles were prepared as controls.

\section{Particle recovery}

Oil-dispersed alginate particles were recovered through an extraction medium ${ }^{20}$ (protocol A) consisting of acetate buffer solution ( $\mathrm{pH} 4.5$ ) (USP 34) with dehydrating solvents (acetone, isopropanol, and hexane) added under continual stirring $(600 \mathrm{rpm})$ followed by centrifugation $(12,500 \times \mathrm{g}$ at $4{ }^{\circ} \mathrm{C}$ for 10 minutes). Different extraction protocols (B-F) were developed, which can be seen in Table 1.

The other protocols included a first step where the oil-dispersed particles were submitted to extraction with hexane (protocols B-E) or a hexane/acetate buffer mixture (protocol F) for 1 hour in an orbital shaker. Particle recovery occurred after the addition of the other solvents by decantation. Different volume ratios of dehydrating solvents with acetate buffer were studied. Recovery yield was determined by measuring insulin content in the aqueous phase after particle recovery (Equation 1):

$$
\text { Recovery yield }(\%)=\frac{\text { Insulin in the aqueous phase }}{\text { Total amount of insulin }} \times 100
$$

Before emulsification, the oil phase was stained with Nile red to better assess the efficiency of oil elimination from particles.

Added solvents to recover particles were removed by using a rotoevaporator system (BUCHI, V-700, Vacuum controller V-870/B-491/R-210) under reduced pressure at controlled temperature.

\section{Ultrasonication exposure}

Ultrasonication was applied to the aqueous suspension of particles with an ultrasonicator (Sonics ${ }^{\circledR}$, VCX130; Sonics \& Materials, Newton, CT, USA) with an amplitude of $60 \%$ for 5 and 10 minutes in order to break up agglomerates. The temperature was kept below $25^{\circ} \mathrm{C}$ by using an ice bath.

\section{Determination of insulin content}

Insulin was extracted from particles through dissolution in sodium citrate $(55 \mathrm{mM})$ prepared in phosphate buffer solution (PBS) at pH 7.4 (USP 34) for 1 hour in an orbital shaker. After particle dissolution, the medium was centrifuged $(12,500 \times \mathrm{g}$ at $4{ }^{\circ} \mathrm{C}$ for 10 minutes) and insulin content in supernatant was assessed by HPLC.

\section{Emulsion and particle size}

Emulsion droplet size measurements were performed by dynamic light scattering (DLS) (DelsaNano C, Beckman Coulter Delsa ${ }^{\mathrm{TM}}$, Krefeld, Germany). Measurements were carried out at $25^{\circ} \mathrm{C}$ with a detection angle of $60^{\circ}$. The size distribution was represented by normalized intensity

Table I Composition of protocol media to recover emulsion-dispersed particles

\begin{tabular}{|c|c|c|c|c|c|c|}
\hline \multirow[t]{2}{*}{ Protocol } & \multicolumn{6}{|c|}{ Emulsion/solvent volume ratio } \\
\hline & Hexane & Acetate buffer pH 4.5 & Methanol & Isopropanol & Acetone & Ethanol \\
\hline A & $\mathrm{I}: 0.04$ & $\mathrm{I}: 0.56$ & - & $\mathrm{I}: 0.12$ & $\mathrm{I}: 0.16$ & - \\
\hline$B$ & I:I.50 & - & $\mathrm{I}: 3$ & $\mathrm{I}: 2.00$ & - & - \\
\hline C & $\mathrm{I}: 1.50$ & - & $\mathrm{I}: 3$ & - & $\mathrm{I}: 2.00$ & - \\
\hline D & $\mathrm{I}: \mathrm{I} .50$ & - & $\mathrm{I}: 5$ & - & - & - \\
\hline$E$ & $\mathrm{I}: 0.50$ & - & - & $\mathrm{I}: 0.70$ & - & $\mathrm{I}: 1.0$ \\
\hline $\mathrm{F}$ & $\mathrm{I}: 0.15$ & $\mathrm{I}: 1.25$ & - & $\mathrm{I}: 0.20$ & - & $\mathrm{I}: 0.3$ \\
\hline
\end{tabular}

Notes: The solvents included in protocol A were added simultaneously and particles were separated by centrifugation $\left(12,500 \times \mathrm{g}\right.$ at $4{ }^{\circ} \mathrm{C}$ for 10 minutes); the other protocols began with dissolution in hexane (protocols B-E) or hexane/acetate buffer $\mathrm{pH} 4.5$ (protocol F) for I hour before the addition of the other solvents and particles were separated by decantation. 
distribution. The instrument was routinely checked and calibrated using a standard latex particle kit (Beckman Coulter, Inc., Krefeld, Germany).

Particle-size distribution analysis was performed by laser diffraction (LS) using a particle size analyzer (Beckman Coulter $^{\circledR}$ LS 13 320; Beckman Coulter, Inc., Miami, FL, USA) with polarization intensity differential scattering. The real and the imaginary refractive index were set to 1.36 and 0.01 , respectively. Three measurements of 90 seconds were used and the size distribution was represented by volume, given as diameter values corresponding to percentiles of $10 \%, 50 \%$, and $90 \%$ together with the relative span value. Particle size before and after each cycle of ultrasonication was additionally investigated by DLS.

\section{Zeta potential}

Surface charge was determined by electrophoretic light scattering using a zeta potential analyzer (DelsaNano C, Beckman Coulter Delsa ${ }^{\mathrm{TM}}$ ) at $\mathrm{pH} 4.5$ and $25^{\circ} \mathrm{C}$. The equipment was routinely checked and calibrated using mobility standard (Beckman Coulter, Inc.).

\section{Insulin EE}

Insulin EE was calculated by the difference between the total amount of insulin used to prepare particles and the amount of free insulin per total amount of insulin. Insulin-loaded particles were separated from aqueous supernatant containing free insulin by centrifugation $\left(12,500 \times g\right.$ at $4^{\circ} \mathrm{C}$ for 10 minutes), and the amount of free insulin was determined by HPLC.

\section{Morphological analysis}

Morphology of single surfactant-assisted particles was monitored by scanning electron microscopy (SEM). The suspension was diluted in deionized water (Milli-Q) $(1: 10, \mathrm{v} / \mathrm{v})$ and dried in a desiccator for 24 hours. Particle morphology was observed using a scanning electron microscope (Phenom G2 Pure). The shape, surface, and mass spectroscopy of cosurfactant and ultrasonication-assisted particles were analyzed by field environmental-cryo-SEM scanning microscope (EDSJEOL JSM 6301 F, Oxford INCA Energy 350, Gatan Alto 2500). Phosphotungstic acid-stained particles were frozen using liquid nitrogen slush $\left(-210^{\circ} \mathrm{C}\right)$ under vacuum to allow their fracture in order to obtain a fresh and clean surface for examination. Then, samples were sublimated at $-90^{\circ} \mathrm{C}$ for 4 minutes to remove top layers of water molecules. Finally, samples were sputter coated with gold/palladium for 40 seconds, followed by image capturing.

\section{Insulin retention/release studies}

For determination of the insulin retention/release profile in enzyme-free simulated digestive fluids, $5 \mathrm{~mL}$ of aqueous suspension of particles were incubated in $10 \mathrm{~mL}$ of simulated gastric fluid without pepsin (USP 34 ) at $37^{\circ} \mathrm{C}$ for 2 hours with shaking of 100 strokes/minute using a shaking water bath (SS40-D; GRANT, Cambridge, UK), followed by incubation of particles in $10 \mathrm{~mL}$ of simulated intestinal fluid without pancreatin (USP 34) for 6 hours. Sample aliquots were collected and replaced by the same volume of fresh incubation medium at predetermined times. For determination of insulin released from particles, withdrawn samples were centrifuged at $12,500 \times g$ at $4{ }^{\circ} \mathrm{C}$ for 10 minutes before HPLC injection. The difference between the initial amount of insulin and the insulin in the supernatant corresponds to the amount of insulin retained within particles.

\section{Insulin quantification}

Insulin was analyzed by HPLC (LC-2010 HT; Shimadzu Co., Kyoto, Japan) equipped with a quaternary pump, a column $5 \mu \mathrm{m}, 4.6 \times 250 \mathrm{~mm}$ (X-Terra RP 18; Waters Co., Milford, MA, USA), and precolumn $5 \mu \mathrm{m}, 4 \times 4 \mathrm{~mm}$ (Purospher STAR RP-18; Merck KGaA, Darmstadt, Germany). The mobile phase consisted of acetonitrile and $0.1 \%$ trifluoroacetic acid aqueous solution initially set in the ratio of $30: 70(\mathrm{v} / \mathrm{v})$, which was linearly changed to 40:60 (v/v) over 5 minutes. From 5 to 10 minutes, the ratio of 40:60 $(\mathrm{v} / \mathrm{v})$ was kept constant. Eluent was pumped at a flow rate of $1 \mathrm{~mL} / \mathrm{min}$, the injection volume was $20 \mu \mathrm{L}$, and detection wavelength was $214 \mathrm{~nm}$. The chromatograms were recorded and the peak area responses were measured using an automatic integrator. ${ }^{37}$

\section{Quantum mechanical studies}

The three-dimensional structures of sodium alginate and dextran sulfate were constructed using a software program (Spartan'10 v1.1.0; Wavefunction, Inc., Irvine, CA, USA). Each structure was submitted to energy minimization to obtain the most energetically stable conformation. Then, geometry optimization by the PM6 semiempirical method was conducted and the stereoelectronic properties were obtained, such as the frontier molecular orbitals (HOMO and LUMO) distribution coefficient, and energy, dipole moment vector, and volume. Quantum chemical calculations for other polymers were also performed, such as cellulose sulfate, ${ }^{38}$ sodium polyphosphate, ${ }^{39}$ and sodium carboxymethylcellulose, ${ }^{40}$ previously described as reinforcement polymers. 


\section{Conformational stability of insulin}

\section{Circular dichroism spectroscopy}

To evaluate the conformational stability of insulin released from particles, circular dichroism (CD) spectroscopy was performed with a spectropolarimeter (Olis DSM 20 CD; Online Instrument Systems, Bogart, GA, USA) equipped with a temperature controller, continuously purged with nitrogen, and controlled by GLOBALWORKS software. Spectra were collected at $37^{\circ} \mathrm{C}$ using a $0.1 \mathrm{~cm}$ cell over the wavelength range of 200-260 nm. A scanning speed $(7 \mathrm{~nm} / \mathrm{min})$ with a 6-second response time was employed. Each spectrum represents an average of three consecutive scans, and blank buffer subtraction and data analysis were performed. The molar ellipticity of insulin was calculated as:

CD signal $\times$ mean residual weight of each insulin residue/insulin concentration

where insulin concentration is $\mathrm{mg} / \mathrm{mL} \times$ cell pathlength $(0.1 \mathrm{~cm})$. The concentration of insulin was determined by UV absorption at $276 \mathrm{~nm}$, using a molar extinction coefficient of $6,070 \mathrm{M}^{-1} \cdot \mathrm{cm}^{-1}$. Samples for CD analysis were prepared by dissolution in PBS $\mathrm{pH}$ 7.4. The spectra of insulin released from nanoparticles collected before and after rotoevaporation and ultrasonication were compared with that of nonencapsulated insulin solution made in PBS pH 7.4 at a similar concentration. The secondary structure components ( $\alpha$-helix, $\beta$-sheet, turn, and random coil) of insulin samples were estimated through the CDSSTR algorithm with reference set 7 from the DICHROWEB website (http:// dichroweb.cryst.bbk.ac.uk/html/home.shtml). ${ }^{41}$

\section{Fourier transform infrared spectroscopy analysis}

Nonencapsulated insulin in solution before and after ultrasonication and insulin entrapped into alginate/dextran sulfate particles before and after ultrasonication were analyzed through Fourier transform infrared spectroscopy (FTIR) technique. All spectra were collected with 256 scans and a $4 \mathrm{~cm}^{-1}$ resolution in the region of 4,000-6,00 $\mathrm{cm}^{-1}$. Insulin spectra were obtained following a double subtraction procedure, ${ }^{42}$ followed by a 15-point Savitsky-Golay second derivative, and a baseline correction using a 3- to 4-point adjustment at the amide I region $\left(1,710-1,590 \mathrm{~cm}^{-1}\right)$. Finally, all spectra were area-normalized for further comparison. All of the described spectral treatment was performed using the HorizonMB FTIR software (ABB, Zurich, Switzerland) ${ }^{43}$

\section{Insulin biological activity}

Animals were maintained and tested in accordance with the Federation of European Laboratory Animal Science
Association and the European Union Council Directive 86/609/EEC. Animals procedures and manipulations were conducted according to the ethical principles and guidelines for housing and experiments on animals of the Direcção Geral de Veterinária. Male Wistar rats weighing 250-350 g were housed under controlled temperature and humidity, and a 12-12-hour light-dark cycle. Diabetes was induced with single intraperitoneal injection of $60 \mathrm{mg} / \mathrm{kg}$ streptozotocin in citrate buffer $\mathrm{pH} 4.5$ to damage the pancreatic cells. During the 24 hours after streptozotocin injection, rats were given $5 \%$ glucose physiologic solution to prevent hypoglycemia due to pancreatic damage. After 3 days, rats with frequent urination, loss of weight, and fasting plasma glucose levels higher than $250 \mathrm{mg} / \mathrm{dL}$ were randomized into three groups (six rats per group) for in vivo studies. The animals were fasted for 12 hours before the experiments with free access to water. To minimize the diurnal blood glucose fluctuations, experiments were performed during the 12-hour light cycle.

Insulin bioactivity was analyzed in diabetic rats after subcutaneous administration at $2 \mathrm{IU} / \mathrm{kg}$. Animals were tested in two different studies, in which the same groups were used. In the first study, insulin was extracted from nanoparticles through dissolution in sodium citrate $(55 \mathrm{mM})$ prepared in PBS at $\mathrm{pH}$ 7.4. Unloaded particles were submitted to the same treatment and were used as negative control. Nonencapsulated insulin was used as positive control. Two days after the first administration, the second study was carried out with the formulations dispersed in water. Then, insulin entrapped in nanoparticles was tested, and unloaded nanoparticles and nonencapsulated insulin were used as negative and positive controls, respectively.

Blood samples were collected from the tip of the tail vein during an 8-hour span and blood glucose levels were measured using the ACCU-CHEK Aviva device.

\section{Statistical analysis}

All the experiments were performed in triplicate and are represented as mean \pm standard deviation. Statistical evaluation was performed with a one-way analysis of variance followed by Bonferroni post hoc test. A $P<0.05$ was taken as the criterion of significance. The level of significance was set at probabilities of $P<0.05, P<0.01$, and $P<0.001$.

\section{Results and discussion}

Several factors could affect the size of alginate/dextran sulfate particles obtained by emulsification, namely stirring speed, 
emulsification time, viscosity of both internal and external phases, the concentration and type of surfactant, and the recovery protocol. ${ }^{44,45}$ For the design and development of insulin-loaded alginate/dextran sulfate particles, additional components and steps were integrated into previously described alginate-based particles obtained by emulsification, and their influence on physicochemical and biological parameters, such as size distribution and insulin EE, were evaluated.

Protein stability during formulation and productdevelopment costs remain major challenges in pilot scale-up of micro- and nanoparticles which need to be addressed at all levels of research and development so a novel technology can be successfully achieved. Thus, probing insulin secondary structure during the nanoencapsulation process was of major concern, and was assessed whenever possible.

\section{Emulsion size analysis}

Submicron size carriers have previously been shown to enable insulin absorption through intestinal membranes. ${ }^{46,47}$ The influence of nanoparticle size is obvious, giving rise to the possibility that larger-sized particles may remain in the Peyer's patches, which are rich in M-cells, and lead to slower action. Smaller nanoparticles, if appropriately charged, can pass through the lymphatic pathway and later enter the systemic circulation. ${ }^{4}$ Therefore, it is admitted that the optimal size for nanoparticles to be taken up by M-cells would be below $1 \mu \mathrm{m},{ }^{48}$ and more precisely, below $200 \mathrm{~nm} .{ }^{49,50}$

Accordingly, the first approach in this study was to reduce the w/o emulsion droplet size to the nanoscale. An insufficient dispersion of phases during emulsification results in large particles with wide size distribution. ${ }^{51}$ The final size of nanoparticles depends on the globule size throughout the emulsification process, ${ }^{52}$ which is mainly determined by the energy input, as well as by the nature of the emulsifying agent/agents. ${ }^{53}$ To verify the influence of these factors on emulsion size distribution, ultrasonication add-on for 15 and 30 minutes and different ultrasonic amplitudes were tested. It was noticed that an increase in ultrasonication time until 15 minutes led to a displacement of the emulsion sizedistribution curve toward lower size (Figure 1), with median diameters decreasing from 1,119 to $442 \mathrm{~nm}$. On the other hand, increasing time of ultrasonication until 30 minutes led to bigger emulsion droplets. According to these results, there was an optimal ultrasonication time of 15 minutes, and beyond it coalescence may become predominant due to the cavitation phenomena. Therefore, the ultimate size would occur when a balance between droplet breakup and recoalescence is assured. ${ }^{54}$ In this work, the optimal condition

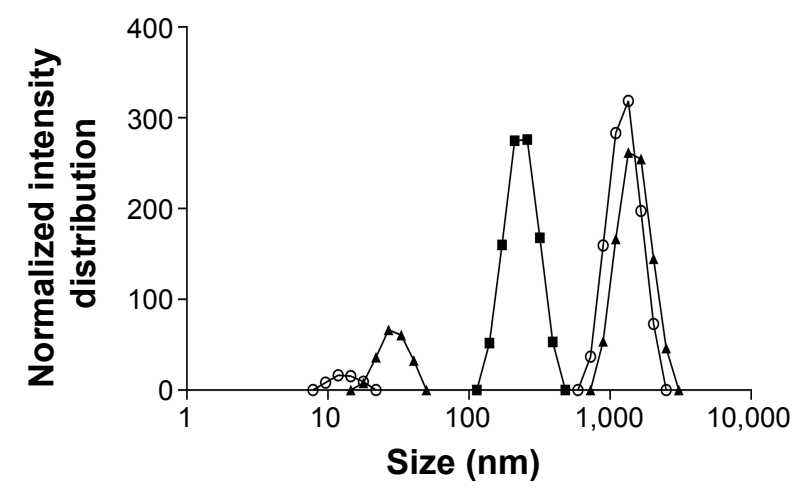

Figure I Influence of ultrasonication time on emulsion size distribution. Notes: Empty circles: without sonication. After 15 minutes (full squares) and 30 minutes (full triangles) of ultrasonication exposure.

was an ultrasonication amplitude of $60 \%$ for 15 minutes. Different ultrasonic amplitudes were tested at 15 minutes of emulsification (data not shown) and the smallest emulsion median diameter was obtained with $60 \%$.

The influence of addition of cosurfactant poloxamer 188 on emulsion size distribution at different time points ranging from 10 to 30 minutes of emulsification was also evaluated (Figure 2). Maintaining the initial percentage of surfactant used, the lowest ratio of sorbitan monooleate/poloxamer 188 required to achieve the necessary HLB to obtain a w/o emulsion $(1.84 \%[\mathrm{v} / \mathrm{v}]$ of sorbitan monooleate and $0.16 \%[\mathrm{w} / \mathrm{v}]$ of poloxamer 188) was employed. There was a decrease in the emulsion size when the cosurfactant was added.

The effect of ultrasonication time in the presence of cosurfactant led to similar results, since periods higher than 15 minutes increased the diameter of emulsion droplets, but with only 10 minutes of exposure, the decrease was less efficient (Figure 2). The use of sorbitan monooleate/poloxamer 188 decreased median diameters of emulsion droplets from

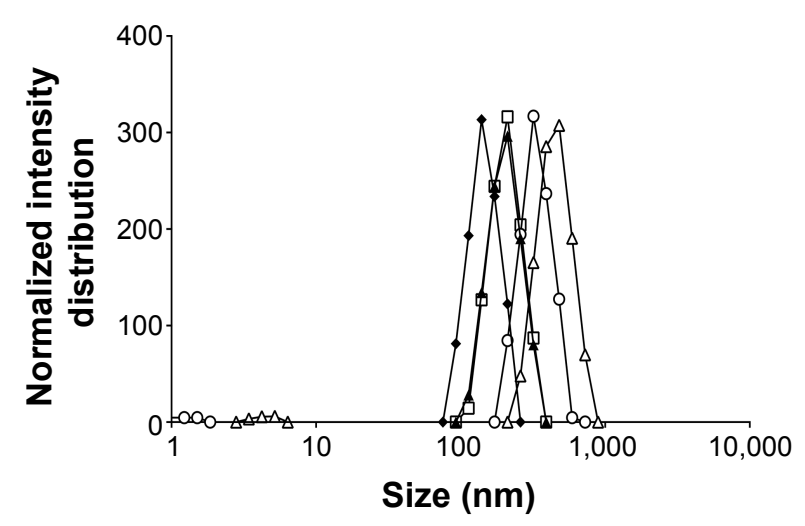

Figure 2 Influence of cosurfactant addition on emulsion size distribution.

Notes: After 10 minutes (empty squares), 15 minutes (full diamonds), 20 minutes (full triangles), 25 minutes (empty circles), and 30 minutes (empty triangles) of ultrasonication exposure. 
442 to $317 \mathrm{~nm}$ after 15 minutes of ultrasonication exposure. This phenomenon can be expected, since a blend of surfactants with high and low HLB are known to produce more stable emulsions than do single surfactants, since they provide better coverage at the interface. ${ }^{55}$ Nanoemulsions are all characterized by a great stability in suspension due to their very small size, essentially due to significant steric stabilization between droplets. ${ }^{56}$

\section{Particle recovery}

The recovery yield of the six protocols studied was evaluated both by its capacity to eliminate residual oil and oil droplets, and by insulin content in the recovered particles. In the standard protocol, ${ }^{20}$ oil-dispersed particles were recovered by a washing step (protocol A, Table 1) associated with centrifugation, which resulted in $29.4 \% \pm 7.2 \%$ (Figure 3 ) of insulin content. Insulin losses could have been due to particle stress during either their preparation or recovery. Firstly, during washing used to remove residual oil from the surface of particles, insulin may have been drawn out, in which smaller particles were probably more affected. Secondly, centrifugal force may have also contributed to insulin losses, due to the porous nature of alginate particles. Thus, the balance between insulin retention inside alginate/dextran sulfate particles, protocol extraction, and/or centrifugal force was considered. Other protocols $(\mathrm{B}-\mathrm{F})$ were designed by varying solvent proportions, and centrifugal force was replaced by gravity separation. Generally, the higher the shear put into creating the emulsion, the finer the droplets produced, and the more stable the emulsion. However, very stable emulsions are more difficult to break. ${ }^{57}$ Therefore, the difficulty to recover oil-dispersed particles to the aqueous suspension

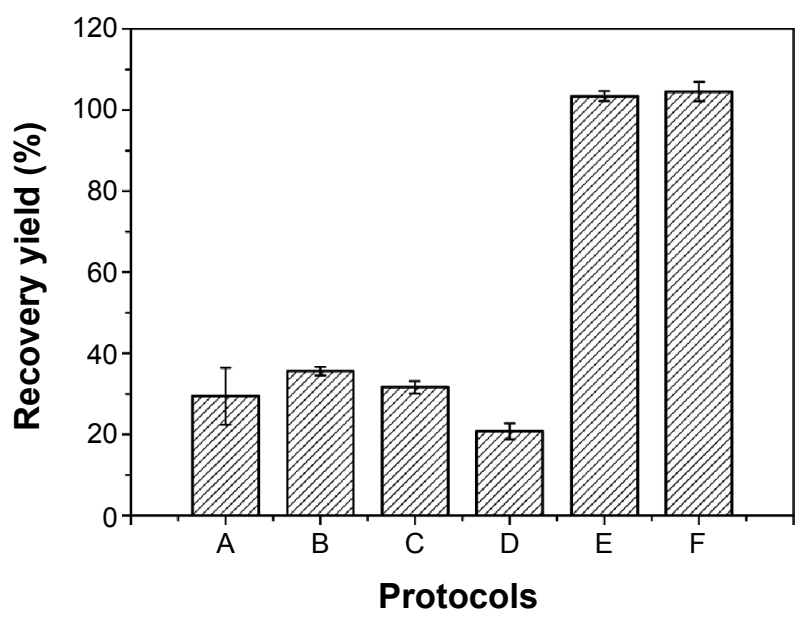

Figure 3 Influence of protocol extractions (A-F) on insulin-based particles recovery yield from the oil phase.

Note: Each value represents mean \pm standard deviation, $n=3$. takes on special importance when lower-sized particles are considered.

All protocols began with emulsion dissolution in hexane for 1 hour, which was considered as the minimum time for complete dissolution. Hexane is often used as a solvent for oil extraction due to its lower boiling point for easy removal after extraction, its nonpolar nature, and its low toxicity when compared to other solvents. ${ }^{58}$ Protocols B-D were also ineffective as recovery media (Figure 3). On the other hand, insulin content after the application of protocol $\mathrm{E}$ was $103.4 \% \pm 1.2 \%$, demonstrating its efficacy. Protocol E was composed of hexane, ethanol, and isopropanol. Ethanol and isopropanol are suitable solvents and have also been used on other nanoparticle formulations. ${ }^{59}$ Bilati et al have shown that the mean particle size was dependent on polarity decrease, ${ }^{60}$ and therefore mixtures of ethanol and isopropanol (less polarsolvent than ethanol) with different proportions were evaluated. In order to minimize the use of these organic solvents, acetate buffer $\mathrm{pH} 4.5$ was included in the washing protocol and protocol $\mathrm{F}$ was chosen, enabling the overall quantification of insulin (Figure 3). The attainment of recovery yields higher than $100 \%$, as happened with particles recovered using protocols $\mathrm{E}$ and $\mathrm{F}$, can be explained by some variations of volumes used during all steps included in particle production and recovery, which may change the theoretical insulin content to values higher or lower than expected. Nile red-stained oil phase allowed for the confirmation of effective oil removal from the aqueous phase, since all the fluorescence was present in the upper phase (oil phase) during decantation.

\section{Removal of organic solvents}

Organic solvents were removed from the extracted particles by rotoevaporation. The best conditions capable of removing all the organic solvents, keeping particles (with the maximum amount of insulin content) in the aqueous phase, were $40^{\circ} \mathrm{C}, 70 \mathrm{mbar}$, for 15 minutes. It is well established that all proteins unfold above their melting temperature. ${ }^{61}$ The melting point described for insulin was $63^{\circ} \mathrm{C} \pm 1^{\circ} \mathrm{C}$, a point where both the size and the intensity start to increase significantly. ${ }^{62}$ Below this temperature, insulin tertiary structure is believed to be stable; ${ }^{37,62}$ therefore, the chosen rotoevaporation temperature would not affect insulin tertiary structure. Furthermore, insulin is unaffected by pressures of 48,000 bar in the solid state at room temperature, which may be due to its relative rigidity combined with its small size. ${ }^{63}$

\section{Particle size analysis}

The presence of aggregates was macroscopically observed after single surfactant-assisted particle recovery. Insulin and 
the biopolymers (both alginate and dextran sulfate) have opposite charges, allowing electrostatic interactions to occur, which may contribute to particle aggregation. ${ }^{64}$

Size distribution of particles is shown in Figure 4 in terms of volume. Diameters of particles corresponding to percentiles of $10 \%, 50 \%$, and $90 \%$ as well as span value are depicted in Table 2. Concerning single surfactant-assisted particles, two different populations can be observed: a small one consisting of nanoparticles and low-sized particles $(<6 \mu \mathrm{m})$; and the main range consisting of higher-sized microparticles $(\mathrm{D} 90=326 \pm 128 \mu \mathrm{m})$. These particles possess, therefore, a wide size distribution, with the predominant percentage being of microparticles, probably due to the presence of aggregates, and a lower percentage of low-sized particles.

Emulsion optimization and recovery-medium development resulted in particles with a median size of $659 \mathrm{~nm}$, with a higher population of particles in the nanometer range, while the higher-sized microparticles, which constituted the main population before, sharply decreased (D90 $=11 \pm 3$ $\mu \mathrm{m})$. The strategies implied in the emulsion optimization (blend of cosurfactants and ultrasonication add-on) were essential to the size reduction observed, since the emulsion droplets produced during the emulsification step originate the final particles. In order to achieve a unique population of nanoparticles, ultrasonication add-on after particle recovery was chosen as a strategy to eliminate or decrease aggregation and therefore to allow a narrower particle-size distribution. Maintaining the same amplitude applied in the emulsion optimization $(60 \%)$, the effect of ultrasonication on particle-size distribution was evaluated and compared for 5 and 10 minutes. After 5 minutes of exposure to ultrasonication, particles showed a deviation to the left of the distribution curve (Figure 4), predominantly to the nanoscale,

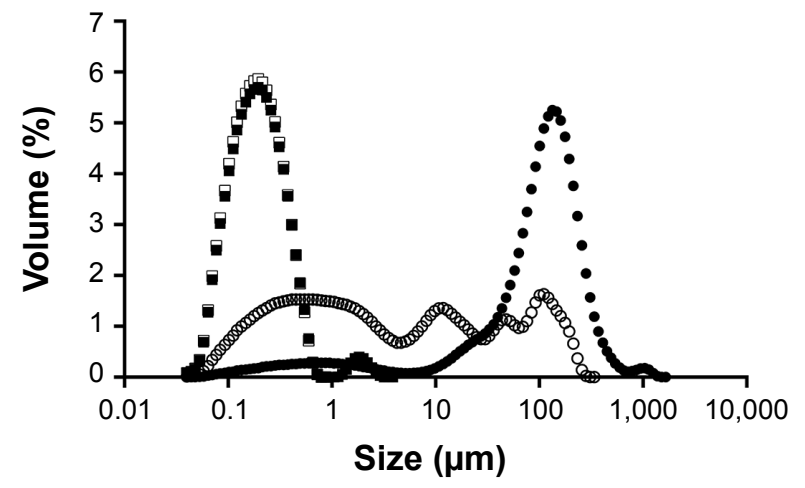

Figure 4 Size distribution of particles under the following processing steps. Notes: Full circles: single surfactant-assisted particles. Empty circles: cosurfactant and ultrasonication-assisted particles. Full squares: after 5 minutes of ultrasonication exposure. Empty squares: after 10 minutes of ultrasonication exposure. with a slight and isolated population of approximately $2 \mu \mathrm{m}$. Consequently, the percentile values suffered a huge decrease, as can be seen in Table 2. After 10 minutes of ultrasonication, on the other hand, a single population of nanoparticles was observed. These particles were also analyzed by DLS. Results are depicted in Table 2, being consistent with the results obtained by LS and confirming the nanoscale size range of particles.

\section{Zeta potential analysis}

The results summarized in Table 3 exhibit that alginate/ dextran sulfate nanoparticles are strongly negatively charged with zeta potentials ranging from -53.4 to $-60.4 \mathrm{mV}$. Negative zeta potential values are essentially due to surface charges of alginate and dextran sulfate chains at $\mathrm{pH} 4.5$, which confer negative charges to nanoparticles. The charge behavior of nanoparticles was also studied at different $\mathrm{pHs,}$ namely at alginate $\mathrm{p} K_{\mathrm{a}}$ and at $\mathrm{pH} 1.5$. At alginate $\mathrm{p} K_{\mathrm{a}}$, alginate chains are almost neutral and the zeta potential of particles increased from -59.6 to $-25.0 \mathrm{mV}$. Particle charge was due to sulfate dextran chains that remained permanently charged since they have a negative first $\mathrm{p} K_{\mathrm{a}}$ value and a second $\mathrm{p} K_{\mathrm{a}}$ value near $2 .{ }^{65}$ At $\mathrm{pH} 1.5$, blocks of dextran sulfate chains become positive and zeta potential increased to $-9.2 \mathrm{mV}$. Thus, nanoparticle charge is essentially due to the matrix of alginate and dextran sulfate, which was proven by the charge behavior of nanoparticles at different $\mathrm{pHs}$. No effect of rotoevaporation and ultrasonication was detected on nanoparticle zeta potential, highlighting the absence of any physical stability changes. Unloaded nanoparticles presented similar results compared to insulin-loaded nanoparticles, demonstrating no effect of insulin regarding particle charge. Zeta potential of insulin-loaded nanoparticles was expected to be less negative compared to unloaded nanoparticles, given the positive charge of insulin at $\mathrm{pH}$ below its isoelectric point: 5.3. The adsorption of insulin on the surface of nanoparticles may be assumed as negligible and insulin was mainly incorporated inside nanoparticles' matrix. Zeta potential of both insulin-loaded and unloaded nanoparticles at $\mathrm{pH} 6.8$ decreased approximately by $10 \mathrm{mV}$ (data not shown), since the matrix of biopolymers became more ionized. The magnitude of zeta potential may indicate the potential electrostatic stability of nanoparticles, with values greater than $30 \mathrm{mV}$ or less than $-30 \mathrm{mV}$ related to nanoparticles with higher stability in suspension and lower aggregation tendency. Particles are strongly charged at $\mathrm{pH} 4.5$, which leads to the prevention of aggregation by repulsive forces upon random impact of adjacent particles, as previously reported. ${ }^{66}$ 
Table 2 Granulometric distribution of particles

\begin{tabular}{|c|c|c|c|c|c|c|}
\hline \multirow[t]{2}{*}{ Formulation } & \multicolumn{4}{|c|}{ Laser diffraction } & \multicolumn{2}{|c|}{ Dynamic light scattering } \\
\hline & $\mathrm{D} \mid 0(\mu \mathrm{m})$ & $\mathrm{D} 50(\mu \mathrm{m})$ & $\mathrm{D} 90(\mu \mathrm{m})$ & Span value & Mean $(\mathrm{nm})$ & PI \\
\hline A & $|4 \pm| \mid$ & $125 \pm 24$ & $326 \pm 128$ & 2.49 & - & - \\
\hline B & $0.134 \pm 0.039$ & $0.659 \pm 0.054$ & $11 \pm 3$ & 16.09 & $556 \pm 12$ & 0.24 \\
\hline B 5-minute ultrasonication & $0.092 \pm 0.056$ & $0.199 \pm 0.439$ & $0.437 \pm 0.320$ & 1.73 & $293 \pm 4$ & 0.30 \\
\hline B I0-minute ultrasonication & $0.091 \pm 0.032$ & $0.194 \pm 0.064$ & $0.407 \pm 0.061$ & 1.63 & $248 \pm 2$ & 0.27 \\
\hline
\end{tabular}

Notes: Particles were prepared with: A) single surfactant; and B) cosurfactant and ultrasonication-assistance. Diameter values (mean \pm standard deviation, $\mathrm{n}=3$ ) corresponding to percentiles of $10 \%, 50 \%$, and $90 \%$, and span value after each processing. Abbreviations: PI, polydispersity index; D, diameter.

\section{Insulin EE}

Insulin EE value was $99.3 \% \pm 0.5 \%$, higher than that previously reported by us. ${ }^{24,67}$ This high level can be attributed to the $\mathrm{pH}$ effect of nanoparticle components. Insulin has an isoelectric point at approximately $\mathrm{pH} 5.3^{68}$ and alginate has $\mathrm{p} K_{\mathrm{a}}$ values of 3.38 and 3.65 for mannuronic and guluronic residues, respectively ${ }^{69}$ Therefore strong electrostatic attractions at the final $\mathrm{pH}$ of 4.5 may occur providing high EE of the oppositely charged protein. ${ }^{24}$ Furthermore, calcium ions interact with the guluronic residues and can establish ionic bridges with negatively charged carboxylic residues of insulin, strengthening the association between insulin and alginate. ${ }^{70}$ The addition of poloxamer 188 can also contribute to insulin EE due to steric stabilization by molecules of poloxamer. ${ }^{66}$

\section{Morphological analysis}

The structure of single surfactant-assisted particles was examined by SEM (Figure 5A). Presence of agglomerates with individual particles of approximately $5 \mu \mathrm{m}$ can be seen.

The structure of the cosurfactant and ultrasonicationassisted nanoparticles was verified by cryo-SEM, which demonstrated good sphericity and smooth surfaces (Figure 5B). The size of nanoparticles determined by morphological analysis is in accordance with the previously measured size (by LS and DLS), lying in the nanoscale range. The smooth and dense particle surface could be due to residual surfactants, as observed previously. ${ }^{71}$
Although both particles demonstrated spherical shape, Figure 5B shows smaller particles with smoother surfaces. These particles were obtained from a more stable emulsion produced with cosurfactant and ultrasonication addition, which originated nanodroplets during emulsification. Small particles are known to dramatically enhance Brownian motion, which further prevents particle aggregation, offers increased stability, ${ }^{72}$ and justifies the lower tendency of cosurfactant and ultrasonication-assisted particles to aggregate compared to single surfactant-assisted particles. Particles of Figure $5 \mathrm{~A}$ have a more porous texture, contrary to particles of Figure $5 \mathrm{~B}$, probably because during the recovery step they lost a significant amount of water during centrifugation, leading to a change of the polymeric structure. ${ }^{20}$ Furthermore, cosurfactant addition has been shown to make particles smoother and slightly porous in previous studies. ${ }^{73}$

\section{Insulin retention/release studies}

Insulin release from nanoparticles was studied as a function of time in enzyme-free simulated digestive fluids as illustrated in Figure 6. Insulin-loaded nanoparticles showed the protective effect of biopolymers at the beginning in simulated gastric fluid during the first 2 hours. This retention capacity can be attributed to the gel strength of nanoparticles in the dissolution media, preventing the release of insulin from the formulation. ${ }^{74}$ Then, a burst release of insulin was noticed under basic conditions ( $\mathrm{pH}$ 6.8) simulating the intestinal environment. In this $\mathrm{pH}$, polymers swell due to deprotonation, ${ }^{75}$ allowing insulin release from nanoparticles. Total insulin

Table 3 Zeta potential (mean \pm standard deviation, $n=3$ ) of nanoparticles after each processing step at different $\mathrm{pHs}$

\begin{tabular}{llll}
\hline Formulation & $\mathbf{p H ~ 4 . 5}(\mathbf{m V})$ & $\mathbf{p H}=$ alginate $\mathbf{p K}_{\mathrm{a}}(\mathbf{m V})$ & $\mathbf{p H ~} \mathbf{. 5}(\mathbf{m V})$ \\
\hline Insulin-loaded nanoparticles & $-59.6 \pm I I .5$ & $-25.0 \pm 2.8$ & $-9.2 \pm 2.0$ \\
- After rotoevaporation & $-53.4 \pm 6.4$ & $-34.9 \pm 1.5$ & $-11.0 \pm 3.6$ \\
- After ultrasonication & $-60.4 \pm 3.3$ & $-27.6 \pm 0.8$ & $-9.3 \pm 0.7$ \\
Unloaded nanoparticles & $-50.9 \pm 4.6$ & $-29.8 \pm 1.5$ & $-8.0 \pm 0.2$ \\
\hline
\end{tabular}



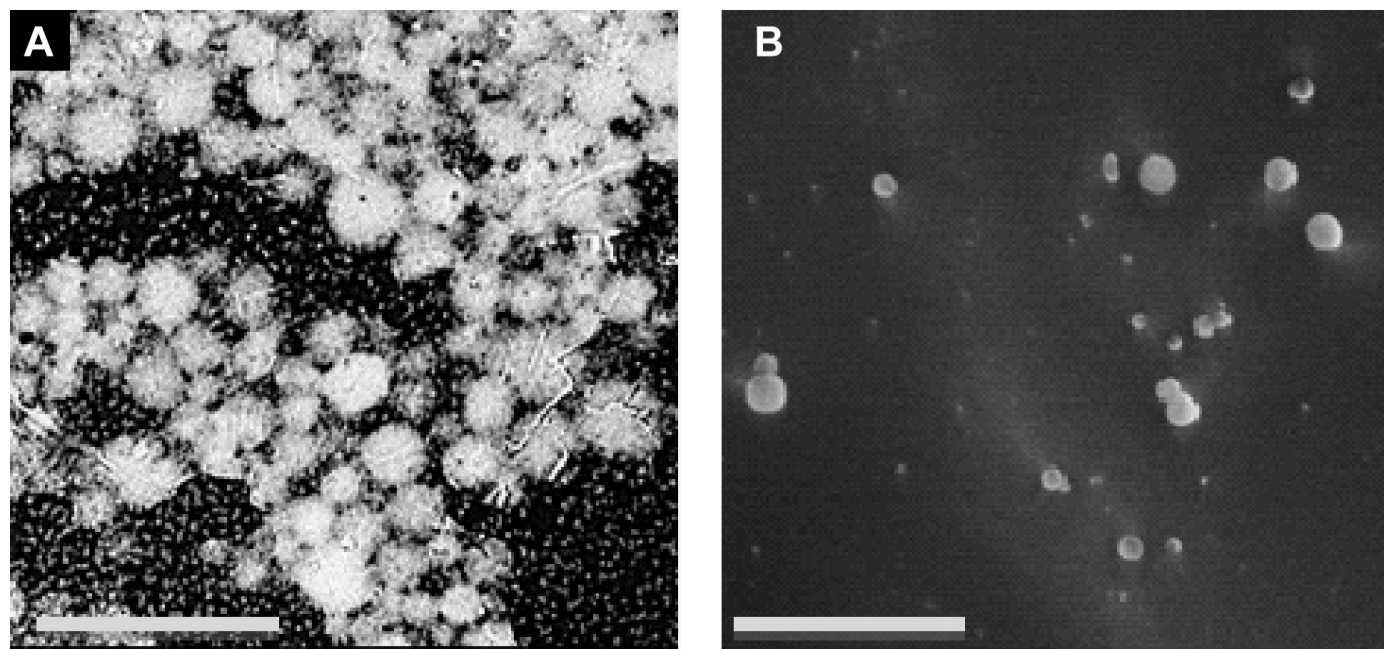

Figure 5 SEM microphotograph.

Notes: (A) Scale bar $=20 \mu \mathrm{m}$; single surfactant-assisted particles. (B) Scale bar $=1 \mu \mathrm{m}$; cryo-SEM microphotographs of cosurfactant and ultrasonication-assisted particles. Abbreviation: SEM, scanning electron microscopy.

release was achieved during the following 3 hours. The release profile of insulin showed a $\mathrm{pH}$-dependent behavior, indicating that these nanoparticles are an effective controlleddelivery system for insulin. Previous studies with biopolymer particles also prepared with emulsification/internal gelation showed a burst release during the first 4 hours but a sustained release profile up to 10 hours, achieving only approximately $75 \%$ of cumulative-released insulin. ${ }^{27}$ Although a sustained release of insulin is desired, insulin release should not take that long, unless particles are sufficiently mucoadhesive to stay in the intestinal epithelium, maintaining insulin structure for such a prolonged period of time.

\section{Quantum mechanical studies}

Theoretical parameter analysis showed a relationship between highest occupied molecular orbital (HOMO) and

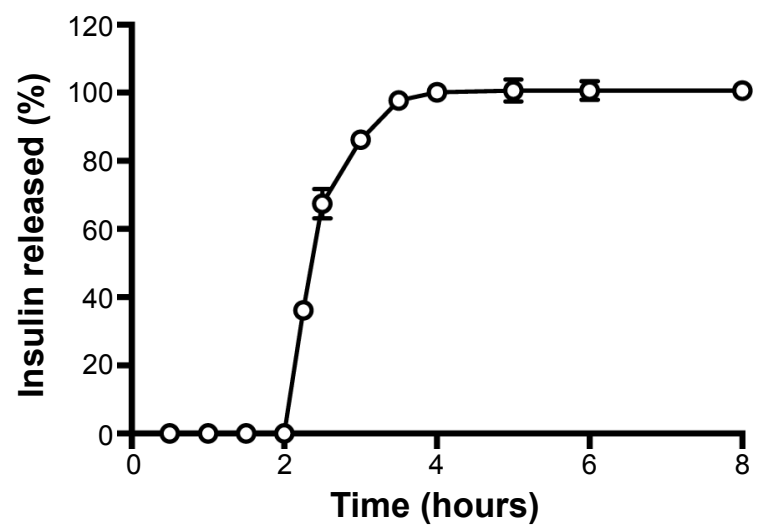

Figure 6 Insulin release profile simulated in $\mathrm{pH} 1.2$ gastric fluid for 2 hours followed by 4 hours in $\mathrm{pH} 6.8$ intestinal fluid at $37^{\circ} \mathrm{C}$.

Note: Each value represents mean \pm standard deviation, $n=3$. lowest unoccupied molecular orbital (LUMO), dipole moment vector, and EE values (Table 4). GAP value is the difference between the energies of the frontier orbitals (HOMO-LUMO) and plays a critical role in chemical reactivity, which is related to intermolecular interactions. ${ }^{76}$ Previous experimental results ${ }^{77}$ revealed that the reinforcement of alginate matrix with dextran sulfate presented the highest insulin EE and one of the lowest particle sizes, compared to the other anionic polymers. Considering theoretical results for the reinforcement polymers, HOMO-LUMO gap (Figure 7A) and dipole moment values of dextran sulfate were the closest to alginate values (Table 4). These findings can be directly related to important electrostatic interactions in nano complex formation and explain the high insulin EE found in our study.

Besides, based on HOMO and LUMO distribution coefficients and dipole moment vector orientation, it was possible to predict the interactions between alginate and dextran sulfate (Figure 7B). Based on these results, it could be seen that the negatively charged groups of alginate remain free

Table 4 Theoretical studies of HOMO-LUMO gap and dipole moment of anionic polymers

\begin{tabular}{lll}
\hline Anionic polymers & $\begin{array}{l}\text { HOMO- } \\
\text { LUMO gap }\end{array}$ & $\begin{array}{l}\text { Dipole moment } \\
\text { (debye) }\end{array}$ \\
\hline Sodium alginate & -9.34 & 16.55 \\
Dextran sulfate & -9.50 & 11.85 \\
Cellulose sulfate & -10.00 & 7.82 \\
Sodium polyphosphate & -11.45 & 5.80 \\
Sodium carboxymethylcellulose & -8.33 & 41.54 \\
\hline
\end{tabular}

Abbreviations: HOMO, highest occupied molecular orbital; LUMO, lowest unoccupied molecular orbital. 

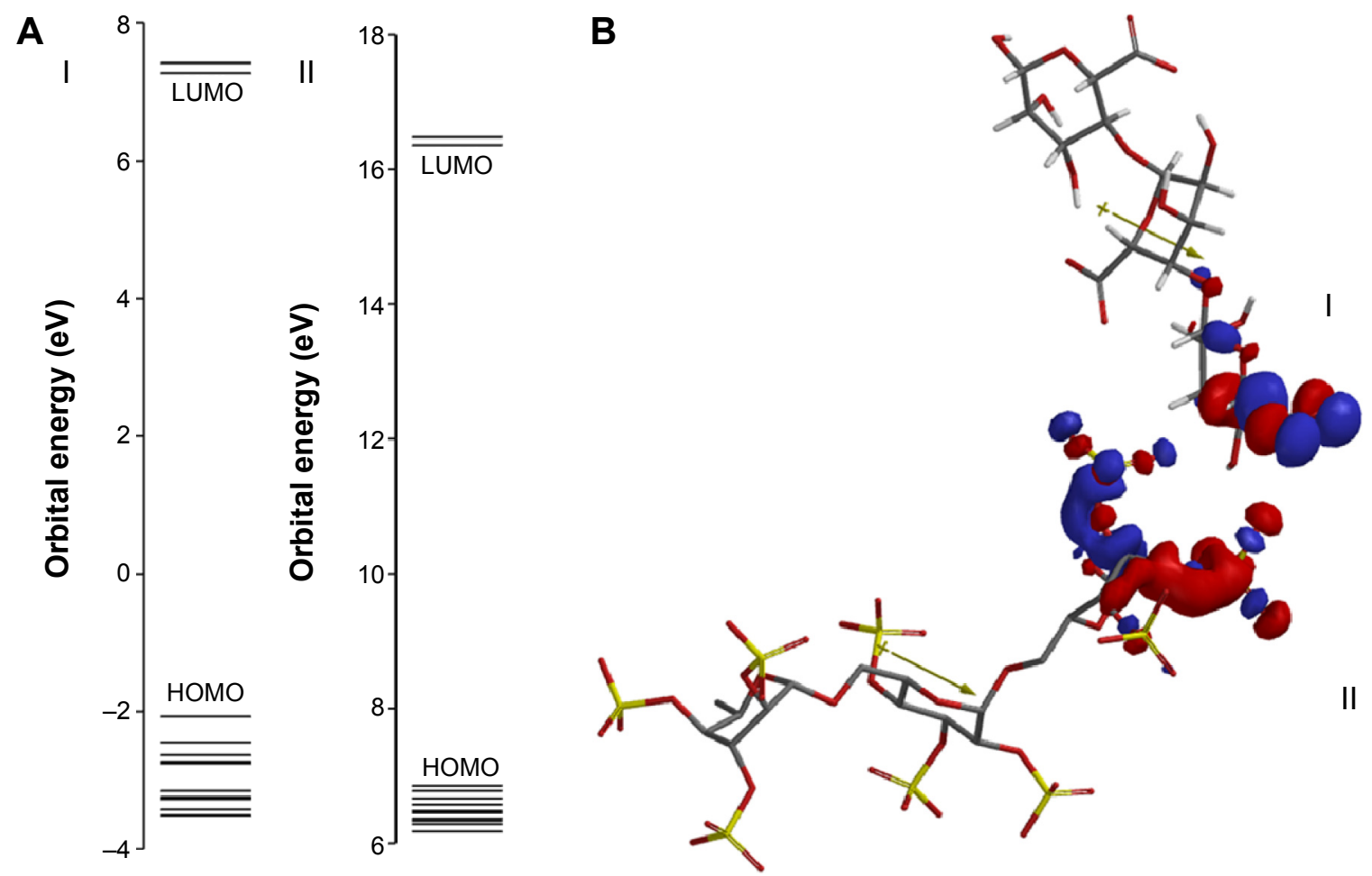

Figure 7 Predicted interaction between biopolymers based on distribution of frontier molecular orbitals (HOMO and LUMO).

Notes: (A) HOMO-LUMO gap and (B) interaction between alginate (I) and dextran sulfate (II) based on their distribution coefficients of HOMO and LUMO, respectively. The positive and negative regions are represented by blue and red colors, respectively.

Abbreviations: HOMO, highest occupied molecular orbital; LUMO, lowest unoccupied molecular orbital.

to establish electrostatic interactions with positively charged groups from the insulin surface. So, based on the HOMO and LUMO distributions coefficients, it was possible to suggest the interaction between the sodium alginate and dextran sulfate. Considering the high value of insulin $\mathrm{EE}(99.3 \% \pm 0.5 \%)$ obtained with these nanoparticles, a correlation between theoretical studies and insulin EE can be predicted.

\section{Conformational stability of insulin}

CD and FTIR are techniques that can provide structural information about proteins. The spectroscopic characterization of protein secondary structure is often partially unreliable when samples are not extremely pure and abundant. This problem may be overcome by the combination of $\mathrm{CD}$ and FTIR. ${ }^{78}$ The stability of insulin encapsulated and released from nanoparticles was evaluated using these two techniques at different time points. Conformational changes in the secondary structure of insulin during each processing of encapsulation were monitored by CD and FTIR analysis.

\section{CD spectroscopy}

The conformation of proteins or peptides is important for the exertion of optimal therapeutic effect and it can be easily damaged under conditions such as high temperature, mechanical manipulation, and exposure to organic solvents. Generally, the secondary structure ( $\alpha$-helix and $\beta$-sheet) can illustrate the efficacy of insulin. CD is regarded as one of the most effective methods to evaluate the secondary structures of proteins and peptides. ${ }^{79}$

Figure 8 represents the CD spectra of insulin secondary structures. Nonencapsulated insulin showed two minima at 210 and $220 \mathrm{~nm}$, which is typical of predominant $\alpha$-helix structure proteins. These results were in agreement with results presented by other studies. ${ }^{70,80} \mathrm{CD}$ spectra of insulin released from nanoparticles after recovery, rotoevaporation, or exposure to ultrasonication revealed no substantial alterations in the two $\alpha$-helix minima when compared to nonencapsulated insulin. To better assess the structural transition, the CD spectra of insulin were de-convoluted through the software from the DICHROWEB website ${ }^{41}$ and the results are displayed in Table 5. Practically no difference in the relative proportions of secondary structure elements was detected along the preparation of nanoparticles, suggesting no insulin fibrillation/aggregation among the conditions tested.

\section{FTIR analysis}

The second-derivative FTIR spectra of insulin are represented in Figure 9. Characteristic bands found in the infrared spectra 


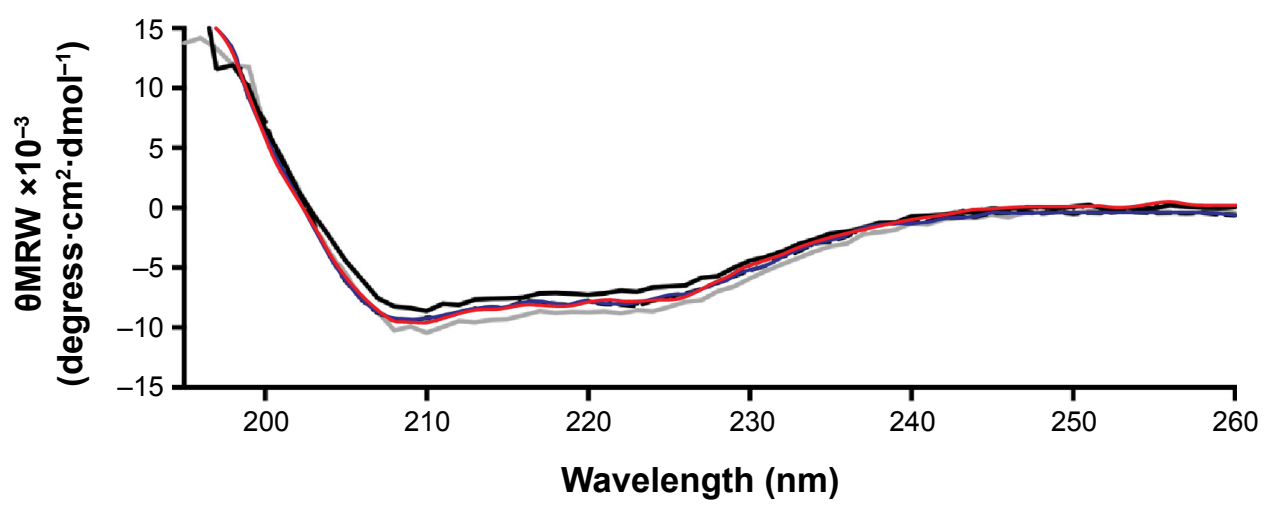

Figure 8 Circular dichroism spectra of insulin in phosphate-buffered saline at $\mathrm{pH} 7.4$ and $25^{\circ} \mathrm{C}$.

Notes: Solid black line: nonencapsulated insulin. Solid blue line: insulin released from nanoparticles after recovery. Solid red line: insulin released from nanoparticles after rotoevaporation. Solid grey line: insulin released from nanoparticles after ultrasonication exposure.

Abbreviation: $\theta M R W$, mean residue molar ellipticity.

of proteins and polypeptides include the amide I and amide II. Amide I absorption arises from the amide bonds that link the amino acids and is directly related to the backbone conformation, with a major contribution from $\mathrm{C}=\mathrm{O}$ stretching vibration and minor contribution from the $\mathrm{C}-\mathrm{N}$ stretching vibration. The major component of the amide I spectrum is the $\alpha$-helix band, whereas the $\beta$-sheet and $\beta$-turn bands contribute less to the secondary structure. ${ }^{81}$ The modifications of $\alpha$-helix are the most characteristic of protein denaturation and consequent loss of activity. Nonencapsulated insulin in solution is dominated by $\alpha$-helix content $\left(1,655 \mathrm{~cm}^{-1}\right)$, but also high- and low-frequency $\beta$-sheet content (1,682 and $\left.1,614 \mathrm{~cm}^{-1}\right)$.

In Figure 9 the spectrum of nonencapsulated insulin in solution submitted to ultrasonication presented an area overlap of 0.92 compared to nonencapsulated insulin without processing, indicating that ultrasonication slightly affects insulin secondary structure. This structural modification motivated by ultrasonication was characterized just by a slight decrease on the $\alpha$-helix band. The spectrum of insulin-loaded nanoparticles submitted to ultrasonication presented an area-overlap of 0.75 compared to nonencapsulated insulin in solution, which was similar to the formulation without ultrasonication (0.76), indicating that the native conformation of insulin changed with either ultrasonication or formulation procedure and those changes were quantitatively similar. Besides similar area-overlap values, it was noticed that after entrapment into nanoparticles, insulin structure changed, originating a decrease of $\alpha$-helix and $\beta$-sheet content. Furthermore, after ultrasonication of insulin-loaded nanoparticles, the structure of insulin changed by decreasing its $\alpha$-helix content at approximately $1,655 \mathrm{~cm}^{-1}$ and increasing $\beta$-sheet at 1,635 $\mathrm{cm}^{-1}$. These modifications are representative of insulin denaturation. The band at $1,700 \mathrm{~cm}^{-1}$ also indicated an unordered structural change. Besides the structural changes of insulin upon entrapment into nanoparticles and ultrasonication, the maintenance of $\alpha$-helix band on the second-derivative amide I FTIR spectra, despite some decrease, still is a good indicator of insulin bioactivity. Additionally, possible structural rearrangements of insulin upon entrapment into nanoparticles may help to mitigate the decrease of insulin bioactivity.

\section{Insulin biological activity}

Blood glucose profiles following subcutaneous injection of insulin to diabetic rats at a dose level of $2 \mathrm{IU} / \mathrm{kg}$ are illustrated in Figures 10 and 11. Insulin released from nanoparticles and nonencapsulated insulin promoted a rapid and intense decrease of glycemia, hitting less than $20 \%$ of the initial

Table 5 Estimation results of the secondary structural content of insulin extracted from nanoparticles before and after recovery, rotoevaporation, and ultrasonication as obtained from circular dichroism spectral analyses

\begin{tabular}{lllll}
\hline $\begin{array}{l}\text { Secondary structure } \\
\text { content }\end{array}$ & \multicolumn{2}{l}{$\begin{array}{l}\text { Nonencapsulated } \\
\text { insulin (\%) }\end{array}$} & \multicolumn{4}{l}{ Insulin released from nanoparticles after } \\
\cline { 3 - 5 } & 39 & Recovery (\%) & Rotoevaporation (\%) & Ultrasonication (\%) \\
\hline$\alpha$ & 31 & 42 & 42 & 39 \\
$\beta$ & 8 & 34 & 31 & 31 \\
Turn & 22 & 8 & 7 & 8 \\
Unordered & 21 & 21 & 22 \\
\hline
\end{tabular}




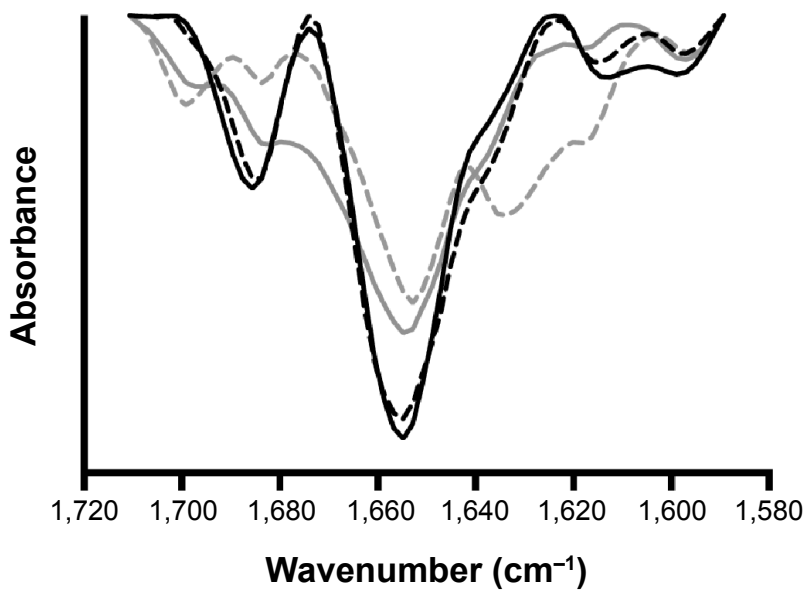

Figure 9 Fourier transform infrared spectra of insulin.

Notes: Fourier transform infrared spectra of nonencapsulated reference insulin in solution (solid black line), nonencapsulated insulin in solution after 10 minutes of ultrasonication exposure (dashed black line), insulin entrapped into nanoparticles (solid grey line), and insulin entrapped into nanoparticles after 10 minutes of ultrasonication exposure (dashed grey line).

values after 2 hours (Figure 10). Then, blood glucose levels increased, reaching approximately $70 \%-90 \%$ of basal values at 8 hours. In the second study (Figure 11), insulin entrapped into nanoparticles showed a slight and less-pronounced decrease of glycemia ( $57 \%$ of the initial values) than insulin released from nanoparticles, whereas nonencapsulated insulin provided a rapid and intense decrease of glycemia, which was, however, also less pronounced than in the first study ( $35 \%$ of the initial values). Then, blood glucose levels increased, reaching approximately $70 \%$ of basal values at 8 hours. Insulin entrapped into nanoparticles allowed a

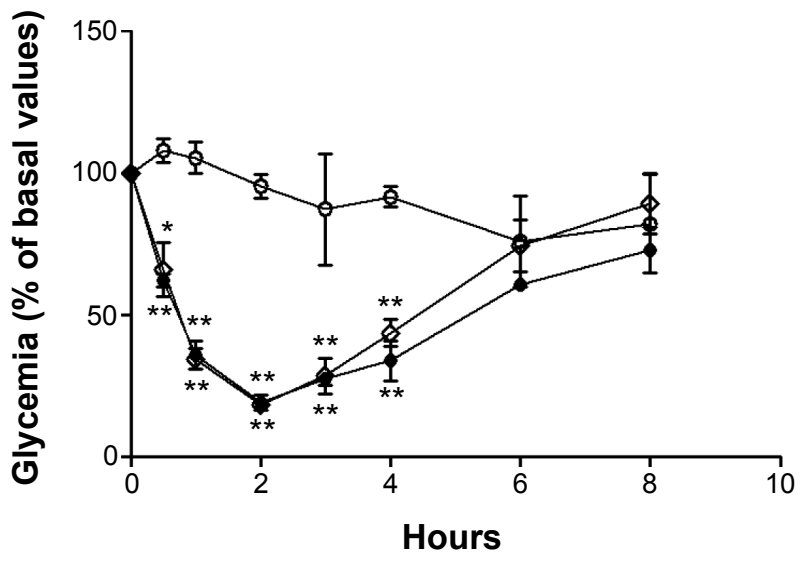

Figure 10 Blood glucose levels of diabetic Wistar rats after subcutaneous administration of insulin.

Notes: Full circles: nonencapsulated insulin. Empty diamonds: insulin released from nanoparticles. Comparison is made to extracted unloaded nanoparticles (empty circles). The formulations were administered in sodium citrate in phosphate-buffered $\mathrm{pH} 7.4$ solution. Results are expressed as mean \pm standard deviation, $\mathrm{n}=6$ per group. Statistically different from empty nanoparticles: $* P<0.5 ; * * P<0.0$ I.

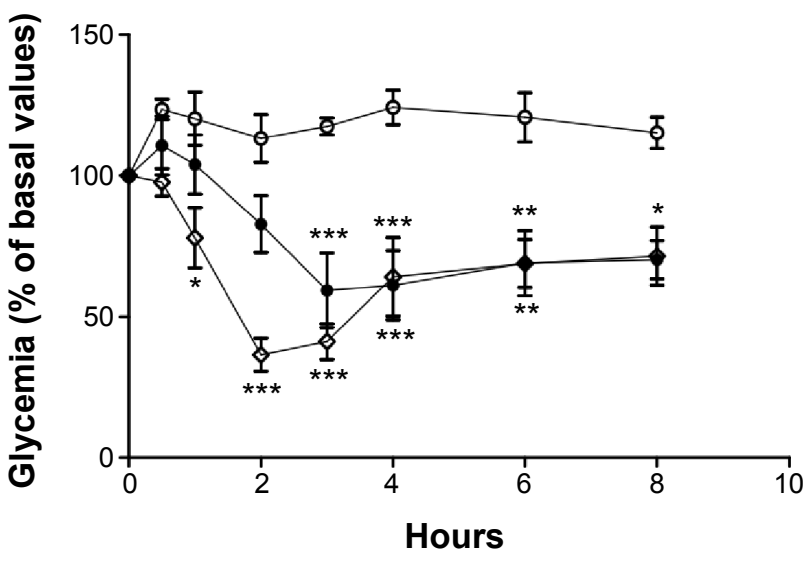

Figure II Blood glucose levels of diabetic Wistar rats after subcutaneous administration of $2 \mathrm{IU} / \mathrm{kg}$ of insulin in water.

Notes: Empty diamonds: nonencapsulated insulin. Full circles: insulin entrapped into nanoparticles. Comparison is made to unloaded nanoparticles (empty circles). The formulations were administered in water. Results are expressed as mean \pm standard deviation, $n=6$ per group. Statistically different from empty nanoparticles: $* P<0.05$; $* * P<0.01 ; * * * P<0.001$.

more controlled and smooth antihyperglycemic effect over time than insulin released from nanoparticles, which was already in the free form (isolated from the polymeric matrix) when subcutaneously administered, enabling a faster and abrupt effect. There were no statistical differences between groups treated with the same dose level of nonencapsulated insulin and insulin-loaded nanoparticles, confirming retention of insulin activity. The integrity of insulin after nanoformulation and, consequently, its biological activity were insured.

\section{Conclusion}

Emulsification/internal gelation technique was successfully optimized to overcome drawbacks imposed by this technique, such as wide size distribution in the microscale and low recovery yield. These results demonstrated that ultrasonication add-on was an efficient strategy that allowed the reduction of the size of emulsion droplets and, therefore, the formation of particles in the nanoscale. This strategy was further improved using a blend of cosurfactants (sorbitan monooleate and poloxamer 188). The recovery of oil-dispersed nanoparticles was also developed, achieving a maximum of insulin content in the aqueous phase. The further submission of these particles to ultrasonication led to a monodisperse population of particles in the nanoscale, observed by the deviation of the size-distribution profile from the micrometric to the nanometric range and the decrease of respective span values.

Molecular modeling studies brought an unprecedented evaluation of quantum mechanics through studies of biopolymers interactions. Results showed a relationship 
between electronic properties and insulin EE. Furthermore, they indicated electronic requirements to improve EE, allowing for prediction of the interaction model between sodium alginate and dextran sulfate.

Strongly negatively charged nanoparticles with high insulin EE and insulin retention within nanoparticles in gastric simulation medium were obtained. The secondary structure of insulin tested by CD showed that insulin retains its biological activity during the process of encapsulation, while FTIR analysis showed rearrangements of insulin structure. However, biological activity of released insulin from nanoparticles after subcutaneous injection to diabetic Wistar rats demonstrated retention of insulin activity. Insulin entrapped into nanoparticles showed a slight and less-pronounced decrease than insulin released from nanoparticles, justified by the interaction of insulin with biopolymers.

This optimized methodology with mild conditions for insulin during encapsulation and easily manipulated procedures allowed for the preparation of alginate/dextran sulfatebased insulin-loaded particles in the nanometric range with retention of insulin activity, which has important applications for the pharmaceutical field.

\section{Acknowledgments}

The authors thank Fundação para a Ciência e Tecnologia (FCT) of Portugal (SFRH/BD/79123/2011) and Coordenação de Aperfeiçoamento de Pessoal de Nível Superior (CAPES) of Brazil.

\section{Disclosure}

The authors report no conflicts of interest in this work.

\section{References}

1. Carino GP, Jacob JS, Mathiowitz E. Nanosphere based oral insulin delivery. J Control Release. 2000;65(1-2):261-269.

2. Fonte P, Araújo F, Silva C, et al. Polymer-based nanoparticles for oral insulin delivery: Revisited approaches. Biotechnol Adv. Epub 2015 Feb 26.

3. Lopes MA, Abrahim BA, Seiça R, Veiga F, Rodrigues CR, Ribeiro AJ. Intestinal uptake of insulin nanoparticles: facts or myths? Curr Pharm Biotechnol. 2014;15(7):629-638.

4. Lopes MA, Abrahim BA, Cabral LM, et al. Intestinal absorption of insulin nanoparticles: contribution of M cells. Nanomedicine. 2014;10(6): 1139-1151.

5. Déat-Lainé E, Hoffart V, Garrait G, Beyssac E. Whey protein and alginate hydrogel microparticles for insulin intestinal absorption: evaluation of permeability enhancement properties on Caco-2 cells. Int J Pharm. 2013;453(2):336-342.

6. Situ W, Chen L, Wang X, Li X. Resistant starch film-coated microparticles for an oral colon-specific polypeptide delivery system and its release behaviors. J Agric Food Chem. 2014;62(16):3599-3609.

7. Du X, Zhang J, Zhang Y, et al. Decanoic acid grafted oligochitosan nanoparticles as a carrier for insulin transport in the gastrointestinal tract. Carbohydr Polym. 2014;111:433-441.
8. Samant M, Banerjee SS, Taneja N, Zope K, Ghogale P, Khandare JJ. Biophysical interactions of polyamidoamine dendrimer coordinated Fe3O4 nanoparticles with insulin. J Biomed Nanotechnol. 2014;10(7): 1286-1293.

9. Sakuma S, Hayashi M, Akashi M. Design of nanoparticles composed of graft copolymers for oral peptide delivery. Adv Drug Deliv Rev. 2001;47(1): 21-37.

10. Soppimath KS, Aminabhavi TM, Kulkarni AR, Rudzinski WE. Biodegradable polymeric nanoparticles as drug delivery devices. J Control Release. 2001;70(1-2):1-20.

11. Hwang WS, Truong PL, Sim SJ. Size-dependent plasmonic responses of single gold nanoparticles for analysis of biorecognition. Anal Biochem. 2012; 421(1):213-218.

12. Hirn S, Semmler-Behnke M, Schleh C, et al. Particle size-dependent and surface charge-dependent biodistribution of gold nanoparticles after intravenous administration. Eur J Pharm Biopharm. 2011;77(3):407-416.

13. Hassani S, Pellequer Y, Lamprecht A. Selective adhesion of nanoparticles to inflamed tissue in gastric ulcers. Pharm Res. 2009;26(5): 1149-1154.

14. Akbar N, Mohamed T, Whitehead D, Azzawi M. Biocompatibility of amorphous silica nanoparticles: Size and charge effect on vascular function, in vitro. Biotechnol Appl Biochem. 2011;58(5):353-362.

15. Cui X, Hunter W, Yang Y, Chen Y, Gan J. Biodegradation of pyrene in sand, silt and clay fractions of sediment. Biodegradation. 2011;22(2): 297-307.

16. Alexakis T, Boadi DK, Quong D, et al. Microencapsulation of DNA within alginate microspheres and crosslinked chitosan membranes for in vivo application. Appl Biochem Biotechnol. 1995;50:93-106.

17. Poncelet D, Lencki R, Beaulieu C, Halle JP, Neufeld RJ, Fournier A. Production of alginate beads by emulsification/internal gelation. I. Methodology. Appl Microbiol Biotechnol. 1992;38(1):39-45.

18. Quong D, Neufeld RJ, Skjåk-Bræk G, Poncelet D. External versus internal source of calcium during the gelation of alginate beads for DNA encapsulation. Biotechnol Bioeng. 1998;57(4):438-446.

19. Ribeiro AJ, Neufeld RJ, Arnaud P, Chaumeil JC. Microencapsulation of lipophilic drugs in chitosan-coated alginatemicrospheres. Int JPharm. 1999; 187(1):115-123.

20. Reis CP, Ribeiro AJ, Neufeld RJ, Veiga F. Alginate microparticles as novel carrier for oral insulin delivery. Biotechnol Bioeng. 2007;96(5): 977-989.

21. Stops F, Fell JT, Collett JH, Martini LG. Floating dosage forms to prolong gastro-retention - the characterisation of calcium alginate beads. Int J Pharm. 2008;350(1-2):301-311.

22. Abbaspour M, Makhmalzadeh BS, Arastoo Z, Jahangiri A, Shiralipour R. Effect of anionic polymers on drug loading and release from clindamycin phosphate solid lipid nanoparticles. Trop J Pharm Res. 2013;12(4): $477-482$.

23. Ma T, Wang L, Yang T, Ma G, Wang S. Homogeneous PLGA-lipid nanoparticle as a promising oral vaccine delivery system for ovalbumin. Asian Journal of Pharmaceutical Sciences. 2014;9(3):129-136.

24. Reis CP, Ribeiro AJ, Houng S, Veiga F, Neufeld R. Nanoparticulate delivery system for insulin: design, characterization and in vitro/in vivo bioactivity. Eur J Pharm Sci. 2007;30(5):392-397.

25. Gaumet M, Gurny R, Delie F. Localization and quantification of biodegradable particles in an intestinal cell model: the influence of particle size. Eur J Pharm Sci. 2009;36(4-5):465-473.

26. Zhang J, Peppard TL, Reineccius GA. Double-layered emulsions as beverage clouding agents. Flavour Fragr J. 2015;30(3):218-223.

27. Santos AC, Cunha J, Veiga F, Cordeiro-da-Silva A, Ribeiro AJ. Ultrasonication of insulin-loaded microgel particles produced by internal gelation: impact on particle's size and insulin bioactivity. Carbohydr Polym. 2013;98(2):1397-1408.

28. Corzo-Martínez M, Soria AC, Villamiel M, Olano A, Harte FM, Moreno FJ. Effect of glycation on sodium caseinate-stabilized emulsions obtained by ultrasound. J Dairy Sci. 2011;94(1):51-58.

29. Kim HY, Han JA, Kweon DK, Park JD, Lim ST. Effect of ultrasonic treatments on nanoparticle preparation of acid-hydrolyzed waxy maize starch. Carbohydr Polym. 2013;93(2):582-588. 
30. Stathopulos PB, Scholz GA, Hwang YM, Rumfeldt JA, Lepock JR, Meiering EM. Sonication of proteins causes formation of aggregates that resemble amyloid. Protein Sci. 2004;13(11):3017-3027.

31. Fonte P, Soares S, Costa A, et al. Effect of cryoprotectants on the porosity and stability of insulin-loaded PLGA nanoparticles after freeze-drying. Biomatter. 2012;2(4):329-339.

32. Sah H. Protein behavior at the water/methylene chloride interface. J Pharm Sci. 1999;88(12):1320-1325.

33. Morlock M, Koll H, Winter G, Kissel T. Microencapsulation of rherythropoietin, using biodegradable poly $\left({ }_{D, L}\right.$-lactide-co-glycolide): protein stability and the effects of stabilizing excipients. Eur J Pharm Biopharm. 1997;43(1):29-36.

34. Kang F, Singh J. Conformational stability of a model protein (bovine serum albumin) during primary emulsification process of PLGA microspheres synthesis. Int J Pharm. 2003;260(1):149-156.

35. Andreas K, Zehbe R, Kazubek M, et al. Biodegradable insulin-loaded PLGA microspheres fabricated by three different emulsification techniques: investigation for cartilage tissue engineering. Acta Biomater. 2011;7(4):1485-1495.

36. ICI Americas Inc. The HLB System: A Time-saving Guide to Emulsifier Selection. Wilmington: ICI Americas Inc.; 1984.

37. Sarmento B, Martins S, Ribeiro A, Veiga F, Neufeld R, Ferreira D. Development and comparison of different nanoparticulate polyelectrolyte complexes as insulin carriers. Int J Pept Res Ther. 2006;12 131-138.

38. Canaple L, Rehor A, Hunkeler D. Improving cell encapsulation through size control. J Biomater Sci Polym Ed. 2002;13(7):783-796.

39. Timmy SA, Victor SP, Sharma CP, Kumari JV. Betacyclodextrin complexed insulin loaded alginate microspheres - oral insulin delivery. Trends Biomater Artif Organs. 2002;15(2):48-53.

40. Gürsoy A, Karakuş D, Okar I. Polymers for sustained release formulations of dipyridamole-alginate microspheres and tabletted microspheres. J Microencapsul. 1999;16(4):439-452.

41. Whitmore L, Wallace BA. DICHROWEB, an online server for protein secondary structure analyses from circular dichroism spectroscopic data. Nucleic Acids Res. 2004;32(Web Server issue):W668-W673.

42. Dong A, Huang P, Caughey WS. Protein secondary structures in water from second-derivative amide I infrared spectra. Biochemistry. 1990;29(13):3303-3308

43. Fonte P, Soares S, Sousa F, et al. Stability study perspective of the effect of freeze-drying using cryoprotectants on the structure of insulin loaded into PLGA nanoparticles. Biomacromolecules. 2014;15(10):3753-3765.

44. Dâas A, Hamdaoui O. Extraction of anionic dye from aqueous solutions by emulsion liquid membrane. J Hazard Mater. 2010;178(1-3): 973-981.

45. Chaouchi S, Hamdaoui O. Acetaminophen extraction by emulsion liquid membrane using Aliquat 336 as extractant. Sep PurifTechnol. 2014;129: $32-40$.

46. Aboubakar M, Couvreur P, Pinto-Alphandary H, et al. Insulin-loaded nanocapsules for oral administration: In vitro and in vivo investigation. Drug Dev Res. 2000;49(2):109-117.

47. Woitiski CB, Sarmento B, Carvalho RA, Neufeld RJ, Veiga F. Facilitated nanoscale delivery of insulin across intestinal membrane models. Int J Pharm. 2011;412(1-2):123-131.

48. Gullberg E, Leonard M, Karlsson J, et al. Expression of specific markers and particle transport in a new human intestinal M-cell model. Biochem Biophys Res Commun. 2000;279(3):808-813.

49. Florence AT. Nanoparticle uptake by the oral route: Fulfilling its potential? Drug Discov Today Technol. 2005;2(1):75-81.

50. des Rieux A, Fievez V, Garinot M, Schneider YJ, Préat V. Nanoparticles as potential oral delivery systems of proteins and vaccines: a mechanistic approach. J Control Release. 2006;116(1):1-27.

51. Silva CM, Ribeiro AJ, Figueiredo M, Ferreira D, Veiga F. Microencapsulation of hemoglobin in chitosan-coated alginate microspheres prepared by emulsification/internal gelation. AAPS J. 2006;7(4):E903-E913.

52. Mainardes RM, Evangelista RC. PLGA nanoparticles containing praziquantel: effect of formulation variables on size distribution. Int $J$ Pharm. 2005;290(1-2):137-144.
53. Dickinson E. Interfacial interactions and the stability of oil-in-water emulsions. Pure and Applied Chemistry. 1992;64:1721-1724.

54. Wu ZM, Zhou L, Guo XD, et al. HP55-coated capsule containing PLGA/RS nanoparticles for oral delivery of insulin. Int $J$ Pharm. 2012;425(1-2):1-8.

55. Attwood D, Florence AT. FASTtrack: Physical Pharmacy. 2nd ed. Pharmaceutical Press; 2008.

56. Anton N, Benoit JP, Saulnier P. Design and production of nanoparticles formulated from nano-emulsion templates-a review. J Control Release. 2008;128(3):185-199.

57. Alvarado V, Wang X, Moradi M. Stability proxies for water-in-oil emulsions and implications in aqueous-based enhanced oil recovery. Energies. 2011;4:1058-1086.

58. Nwabanne JT. Kinetics and thermodynamics study of oil extraction from fluted pumpkin seed. International Journal of Multidisciplinary Sciences and Engineering. 2012;3(6):11-15.

59. Schubert MA, Müller-Goymann CC. Solvent injection as a new approach for manufacturing lipid nanoparticles - evaluation of the method and process parameters. Eur J Pharm Biopharm. 2003;55(1): $125-131$.

60. Bilati U, Allémann E, Doelker E. Development of a nanoprecipitation method intended for the entrapment of hydrophilic drugs into nanoparticles. Eur J Pharm Sci. 2005;24(1):67-75.

61. Wang W. Instability, stabilization, and formulation of liquid protein pharmaceuticals. Int J Pharm. 1999;185(2):129-188.

62. Elsayed A, Remawi MA, Qinna N, Farouk A, Badwan A. Formulation and characterization of an oily-based system for oral delivery of insulin. Eur J Pharm Biopharm. 2009;73(2):269-279.

63. Murphy RB. Anomalous stability of insulin at very high pressure. Experientia. 1978;34(2):188-189.

64. Nguyen VS, Rouxel D, Hadji R, Vincent B, Fort Y. Effect of ultrasonication and dispersion stability on the cluster size of alumina nanoscale particles in aqueous solutions. Ultrason Sonochem. 2011;18(1): 382-388.

65. Reis CP, Ribeiro AJ, Veiga F, Neufeld RJ, Damgé C. Polyelectrolyte biomaterial interactions provide nanoparticulate carrier for oral insulin delivery. Drug Deliv. 2008;15(2):127-139.

66. Woitiski CB, Neufeld RJ, Ribeiro AJ, Veiga F. Colloidal carrier integrating biomaterials for oral insulin delivery: Influence of component formulation on physicochemical and biological parameters. Acta Biomater. 2009;5(7):2475-2484.

67. Pinto Reis C, Neufeld RJ, Ribeiro AJ, Veiga F. Nanoencapsulation I. Methods for preparation of drug-loaded polymeric nanoparticles. Nanomedicine. 2006;2(1):8-21.

68. Chien YW. Human insulin: basic sciences to therapeutic uses. Drug Dev Ind Pharm. 1996;22(8):753-789.

69. Draget KI, Skjåk Bræk G, Smidsrød O. Alginic acid gels: the effect of alginate chemical composition and molecular weight. Carbohydr Polym. 1994;25(1):31-38

70. Sarmento B, Ferreira DC, Jorgensen L, van de Weert M. Probing insulin's secondary structure after entrapment into alginate/chitosan nanoparticles. Eur J Pharm Biopharm. 2007;65(1):10-17.

71. Magenheim B, Benita S. Nanoparticle characterization: a comprehensive physicochemical approach. S.T.P. Pharma Sciences. 1991;1: 221-241.

72. Sakulchaicharoen N, O'Carroll DM, Herrera JE. Enhanced stability and dechlorination activity of pre-synthesis stabilized nanoscale FePd particles. J Contam Hydrol. 2010;118(3-4):117-127.

73. Ahmed MM, El-Rasoul SA, Auda SH, Ibrahim MA. Emulsification/ internal gelation as a method for preparation of diclofenac sodiumsodium alginate microparticles. Saudi Pharm J. 2013;21(1): 61-69.

74. Soni ML, Kumar M, Namdeo KP. Sodium alginate microspheres for extending drug release: formulation and in vitro evaluation. International Journal of Drug Delivery 2. 2010:64-68.

75. George M, Abraham TE. Polyionic hydrocolloids for the intestinal delivery of protein drugs: alginate and chitosan - a review. $J$ Control Release. 2006;114(1):1-14. 
76. Murugavel S, Vijayakumar S, Nagarajan S, Ponnuswamy A. Crystal structure and DFT studies of 4-(1-Benzyk-5-Methyl-1H-1,2,3-Triazol4-YL)-6-(3-Methoxyphenyl)Pyrimidin-2-Amine. Journal of the Chilean Chemical Society. 2014;59:2640-2646.

77. Silva CM, Ribeiro AJ, Ferreira D, Veiga F. Insulin encapsulation in reinforced alginate microspheres prepared by internal gelation. Eur $J$ Pharm Sci. 2006;29(2):148-159.

78. Ascoli GA, Pergami P, Luu KX, et al. Use of CD and FT-IR to determine the secondary structure of purified proteins in the low-microgram range. Enantiomer. 1998;3(4-5):371-381.
79. Pocker Y, Biswas SB. Conformational dynamics of insulin in solution. Circular dichroic studies. Biochemistry. 1980;19(22):5043-5049.

80. Li X, Qi J, Xie Y, et al. Nanoemulsions coated with alginate/chitosan as oral insulin delivery systems: preparation, characterization, and hypoglycemic effect in rats. Int J Nanomedicine. 2013;8:23-32.

81. Nielsen L, Frokjaer S, Carpenter JF, Brange J. Studies of the structure of insulin fibrils by Fourier transform infrared (FTIR) spectroscopy and electron microscopy. J Pharm Sci. 2001;90(1):29-37.

\section{Publish your work in this journal}

The International Journal of Nanomedicine is an international, peerreviewed journal focusing on the application of nanotechnology in diagnostics, therapeutics, and drug delivery systems throughout the biomedical field. This journal is indexed on PubMed Central, MedLine, CAS, SciSearch ${ }^{\circledR}$, Current Contents ${ }^{\circledR} /$ Clinical Medicine,
Journal Citation Reports/Science Edition, EMBase, Scopus and the Elsevier Bibliographic databases. The manuscript management system is completely online and includes a very quick and fair peer-review system, which is all easy to use. Visit http://www.dovepress.com/ testimonials.php to read real quotes from published authors. 